Carnets de géographes

GÉOGRAPHES.

\title{
Inégalités d'accès et coproduction du service d'eau dans les quartiers pauvres de Toamasina (Madagascar)
}

\section{Landitiana Soamarina Miakatra}

\section{OpenEdition}

\section{Journals}

Édition électronique

URL : http://journals.openedition.org/cdg/944

DOI : $10.4000 /$ cdg. 944

ISSN : 2107-7266

Éditeur

UMR 245 - CESSMA

Référence électronique

Landitiana Soamarina Miakatra, «Inégalités d'accès et coproduction du service d'eau dans les quartiers pauvres de Toamasina (Madagascar) », Carnets de géographes [En ligne], 6 | 2013, mis en ligne le 01 septembre 2013, consulté le 24 septembre 2020. URL : http://journals.openedition.org/cdg/ 944 ; DOI : https://doi.org/10.4000/cdg.944

Ce document a été généré automatiquement le 24 septembre 2020.

\section{(i) $९$

La revue Carnets de géographes est mise à disposition selon les termes de la Licence Creative Commons Attribution - Pas d'Utilisation Commerciale - Pas de Modification 4.0 International. 


\title{
Inégalités d'accès et coproduction du service d'eau dans les quartiers pauvres de Toamasina (Madagascar)
}

\author{
Landitiana Soamarina Miakatra
}

1 Depuis les années soixante dix, l'eau potable est devenue un thème majeur des rendezvous internationaux. Elle resurgit régulièrement au cœur des actualités politiques et scientifiques. Seul un malgache sur deux a accès à une source d'eau potable ou protégée. A Toamasina, ville de 200000 habitants située sur la côte est de Madagascar, le premier réseau d'eau date des années trente. L'équipement en eau de la ville est étroitement lié à la présence du port en eau profonde, pour servir les intérêts économiques de la colonie. Il présente également un double objectif : celui de l'hygiène coloniale et celui de la résolution de la croissance urbaine relativement rapide dans les quartiers indigènes. Les quartiers coloniaux bénéficiaient d'un raccordement au réseau d'eau et des bornes fontaines approvisionnaient les quartiers indigènes de Tanambao. L'accès à l'eau était déjà inégal. Compte tenu de l'accroissement de la population et de l'étalement urbain, le service d'eau n'arrive pas à couvrir l'ensemble de la commune urbaine de Toamasina.

2 Cette thèse vise à mieux cerner les conditions d'accès à l'eau potable de la population de la ville de Toamasina et d'identifier les dispositifs de coproduction des services d'eau mis en place dans les quartiers pauvres. Elle privilégie une analyse centrée sur les modalités de gouvernance et de gestion des services, l'implication des acteurs locaux dans l'universalisation de l'eau et de leurs conséquences sur la gestion des quartiers mal lotis.

3 La démarche de recherche s'articule entre la théorie et des études de terrain. Les méthodes de travail à l'appui de cette démarche sont essentiellement qualitatives. Outre l'utilisation d'une bibliographie relativement abondante, elles sont de trois ordres : la collecte de données existantes, la réalisation d'entretiens semi-dirigés auprès des acteurs clés du secteur de l'eau et les enquêtes de terrain focalisées sur les usagers des services d'eau. Les investigations concernent 39 quartiers qui 
correspondent à l'aire d'intervention de différentes associations et Organisations Non Gouvernementales.

4 Après les changements de régimes politiques des années 60-70, le service d'eau est géré par une société d'Etat nommé la Jirama (Jiro sy Rano Malagasy ou Eau et Electricité malgaches). A Toamasina, le réseau public ne dessert que $47 \%$ de la population. La connexion domiciliaire concerne une minorité très largement concentrée dans le centre historique de la ville et quelques quartiers privilégiés qui se trouvent vers le nord. Les bornes fontaines à gestion payante, alimentées par le réseau officiel, permettent la desserte collective des ménages non branchés au réseau. 171 bornes fontaines fonctionnelles sont réparties dans les 5 arrondissements de la commune urbaine de Toamasina.

5 Si les modalités d'accès aux bornes fontaines sont liées aux conditions géographiques du quartier, l'approvisionnement en eau dans certains quartiers peut être assuré par les activités de revente assurées par les abonnés ou le porteurs revendeurs d'eau dans les quartiers sous équipés qui enregistrent souvent une densité de population élevée.

6 Dans les quartiers défavorisés, l'eau souterraine fournit l'essentiel des besoins des ménages. Il s'agit d'un simple système de forage qui consiste à puiser l'eau à l'aide d'une pompe aspirante manuelle. Ce système présente l'avantage d'un service à domicile, la ressource est illimitée, accessible à toute heure, gratuite et les ménages se sentent parfaitement autonomes. Ce système est adapté aux conditions pédologiques du milieu, facile à installer, facile à entretenir et les pièces de rechange sont disponibles localement.

7 L'opérateur du réseau peine à relever le taux d'accès à l'eau potable et cumule au fil des années des pertes techniques et financières résultant du manque de cohérence des politiques de desserte et de financement du renouvellement des réseaux. La politique de gratuité du service des bornes fontaines a été coûteuse pour les communes qui sont dans l'incapacité d'en payer leurs factures. Ces dysfonctionnements ont suscité beaucoup de débats et mettent en cause le monopole d'Etat, le déséquilibre entre offre de service et demande citadine, l'absence d'une politique pour desservir les pauvres et la vétusté des infrastructures sont autant de facteurs qui entravent l'amélioration des cadres de vie de la population.

8 La nouvelle politique de l'eau régi par la loi n 98-029 du 20 janvier 1999 portant code de l'eau définit les principes fondamentaux du service public de l'approvisionnement en eau potable et de l'assainissement limité aux eaux usées domestiques, dans toutes les zones, urbaines comme rurales. Elle s'appuie sur des réformes institutionnelles et la participation du secteur privé et des populations à leur approvisionnement en eau, notamment dans les quartiers pauvres. La participation du secteur privé dans le secteur de l'eau revêt les formes les plus diverses, allant de simples mandats confiés à des entreprises privées dans la cadre de projets, à la promotion globale des échanges et des investissements. Le PPP est sensé améliorer la qualité des services, rentabiliser les investissements et mieux servir les populations.

9 Les réformes entendent responsabiliser les acteurs locaux dans l'harmonisation des actions permettant un développement économique et social. Elles placent les usagers au centre de la réflexion dans le contexte de réforme de service public. Elles consolident dans ce cadre les normes de partenariats entre les parties prenantes dans le but de fournir un service au plus près de la population. Ce dispositif de coproduction apparaît comme un moyen de réconcilier marchandisation et diffusion de l'accès à 
l'eau : en diminuant les coûts de construction puis de maintenance et de gestion des dispositifs d'approvisionnement, la mobilisation des usagers permet de desservir des collectifs urbains pauvres pour lesquels le coût du service conventionnel demeure inaccessible.

La coproduction est une structure partenariale de proximité qui est sensée favoriser l'émergence et la consolidation des normes de coopération entre autorités publiques ou publique-privée et des organisations citadines dans le but de pérenniser des formes de fournitures de services là où les offres conventionnelles sont inadaptés. Plus concrètement, ce système socio technique est analysé dans cette thèse à travers la gestion des points d'eau collectifs à Toamasina.

Dans la plupart des villes malgaches, si les habitants ont longtemps bénéficié d'un accès gratuit aux bornes fontaines ou lavoir public ou bloc douche-WC, considéré comme un service social, à l'avènement du code de l'eau, les premières bornes fontaines à gestion payantes sont apparues dans les quartiers urbains dès les années 2000. Le recouvrement des coûts est assuré par les usagers dans le but de pérenniser le service et de permettre des travaux d'extension des réseaux.

12 L'instauration du paiement du service aux points d'eau collectifs étant formalisée, d'abord à Antananarivo, puis à Mahajanga et Toamasina, l'objectif est de mieux organiser la fourniture de service. Des comités de points d'eau ont été mises en place, assurant la gestion des bornes fontaines. Ces comités émanent d'une association de quartier, du fokontany ou même d'un particulier. La structure de gestion contracte directement un abonnement à la Jirama, perçoit les recettes journalières de la revente d'eau, paie la facture mensuelle à la Jirama et entretient les ouvrages. Une partie des recettes sert à rémunérer le fontainier. Les bornes fontaines sont équipées en compteurs individuels pour faciliter la lecture de leur consommation.

Des avancées sont constatées car on note une amélioration de la desserte en eau dans les quartiers défavorisés. Le nombre de quartiers équipés a augmenté grâce à de nouveaux projets. Par exemple dans le cadre de la phase 2 du programme d'appui aux initiatives des quartiers (PAIQ 2), une trentaine de quartiers ont bénéficié de 28 projets qui portent sur l'accès à l'eau potable, à l'assainissement, à la construction de centres culturels, d'écoles, de centres de santé, de complexes sanitaires, et du désenclavement de quartiers par l'aménagement de ruelles. D'autres projets ont été réalisés par le concours de différentes organisations. Ceci occasionne un accroissement du nombre de points d'eau et celui des ménages bénéficiaires. Le recouvrement des coûts semble avoir atteint ses objectifs car les factures sont payées directement à la Jirama. Le service d'eau contribue de manière significative à l'intégration de ces quartiers dans l'espace urbain et par la même occasion à la responsabilisation des habitants à la gestion de leur cadre de vie.

14 En dépit de ces améliorations, la coproduction du service d'eau présente des limites. Pour certains équipements, il n'est pas rare de voir les usagers "privatiser» des ouvrages pour des usages exclusifs. Cette figure est plus flagrante pour les usagers des lavoirs où les lavandières monopolisent l'ensemble de l'espace. La coproduction est aussi instrumentalisée par certains groupes qui essaient de s'imposer dans la gestion de leur quartier et d'y d'exercer un contrôle. Le secteur de l'eau n'est pas épargné par la corruption et le clientélisme. Ceci se manifeste dans le cadre de financement et dans le choix des quartiers à équiper. Les pouvoirs locaux privilégient souvent leurs avantages. La coproduction entraine également des effets d'aubaine. En effet, les Organisations 
non gouvernementales sont plus attirées vers les mêmes quartiers (déjà équipés) et de ce fait les inégalités entre les quartiers s'accroissent. Il y a des quartiers jugés non rentables du fait de leur éloignement ou de leurs conditions d'accès difficiles.

Après une décennie de mise en œuvre des réformes, le bilan est mitigé. La distribution de l'eau dans les principales villes malgaches est toujours assurée par un opérateur public qui peine à satisfaire les demandes de plus en plus croissantes. La mise en œuvre effective de la législation en vigueur reste encore difficile. Le cadre institutionnel en place est complexe et inachevé et n'a pas permis jusqu'à présent l'implication effective des organisations non étatiques.

Une approche globale du renforcement institutionnel de la maitrise d'ouvrage urbaine permettrait de dépasser ces bilans sectoriels décevants pour avancer dans la voie d'une amélioration des conditions de vie à laquelle aspirent les citadins malgaches.

\author{
Lien électronique \\ www.theses.fr/S67016

\section{Discipline} \\ Géographie

\section{Directeurs} \\ Sylvy Jaglin, Solofo Randrianja \\ Université \\ Université de Nantes
}

Membres du jury de thèse, soutenue le 26 janvier 2012

Athanase Bopda, Professeur, Université du Havre

Sylvy Jaglin, Professeur, Université Paris est Marne La Vallée

François Madoré, Professeur, Université de Nantes

Faranirina Rajaonah, Professeur, Université Paris Diderot

Solofo Randrianja, Professeur, Université de Toamasina, Madagascar

\title{
Situation professionnelle actuelle
}

Membre associé à ESO Nantes UMR 6590. Chercheur à l'Institut d'Etudes Politiques de Madagascar

Courriel de l'auteur

soalandi@gmail.com

\section{INDEX}

Thèmes : Carnets de soutenances 